\title{
INTELLIGENT VISION BASED SNAKE ROBOT
}

\author{
Blessy Mariam Markose ${ }^{1}$, Harshitha Loke ${ }^{2}$ \\ ${ }^{1}$ M.Tech Student, Department of Electronics \& Instrumentation, Hindustan University, Tamil Nadu, India \\ ${ }^{2}$ Assistant Professor, Department of Electronics \& Instrumentation, Hindustan University, Tamil Nadu, India
}

\begin{abstract}
Robots are expected to be new tools for the operations and observations in the extreme environments where humans have difficulties in direct access. One of the important matters to realize mobile robots for extreme environments is to establish systems in their structures which are strong enough to disturbances. Also, while considering surveillance in inaccessible remote areas, a need arises for the presence of a robot capable of intruding into small crevices as well as provides proper surveillance.

This work aims at the implementation of a snake robot for surveillance operations in remote areas. A biologically inspired robot with various motion patterns is taken into consideration. An important problem in the control of locomotion of robots with multiple degrees of freedom is in adapting the locomotors patterns to the properties of the environment. This has been overcome by using control techniques capable of integrating the motion patterns of a snake. Here an attempt is taken to focus on the creeping locomotion of a living snake. In hybrid model, the optimal locomotion of the snake robot is tried to achieve by comparing it with that of a living snake. A wireless real time vision processing is also employed within the robot to improve its performance. The presence of Video acquisition along with processing will be an added advantage for implementation of the robot for highly precise and difficult surveillance applications. Real time processing of video enables proper and efficient control towards obstacle avoidance pattern of the robot. This ensures that the locomotion of the robot is in a bio-inspired highly efficient path towards the target.
\end{abstract}

Keywords: Collision-free behavior, neural oscillator, snake locomotion, steering, real time vision processing

\section{INTRODUCTION}

Biologically inspired robots have been researched and developed in an intense fashion recently. Many researchers expect that the locomotion of living things has high performance in the natural world because of their survival by natural selection. However, when the effectiveness of imitating the biological locomotion is considered, it is important to clarify not only the kinematical and dynamical features but also the principle of the locomotion.

An expected factor is that the knowledge regarding this principle would suggest a more effective motion pattern which is specific to mechanical robots. Practically the great ability of movement of a living snake is useful, for example, in search and rescue missions at disaster sites. In hybrid model, the optimal locomotion of the snake robot is tried to achieve by comparing it with that of a living snake.

Robots are expected to be new tools for the operations and observations in the extreme environments where humans have difficulties in direct access, such as Deep Ocean, space, nuclear plants etc. One of the important matters in realizing of mobile robots for extreme environments is to establish systems in their structures which are strong enough to disturbances. Also, while considering surveillance in inaccessible remote areas, a need arises for the presence of a robot capable of intruding into small crevices as well as provides proper surveillance. This highlights the need and importance of a snake robot.

A hybrid system is proposed for the implementation of snakelike robot. The collision-free behavior for lateral locomotion is tried to achieve by using CPG (Central Pattern Generator) based neural steering method with AMM (Amplitude Modulation Method) steering for head-navigated locomotion. For climbing purposes, Sinus-lifting locomotion has to be analyzed based on constraint forces and energy efficiency for wheel-less system.

\subsection{Objective}

The goal is to demonstrate a collision free behavior of the snake robot through the use of neurally controlled steering. Due to its peculiar structure and $S$-shaped motion pattern, the snake robot cannot turn right or left as easily as wheeled robots can. Hirose proposed the serpenoid curve to model the movement of a snake robot [17], and discussed a steering principle for turn motion by adding a bias value to the joint angles. Such a bias means that the amplitude of the joint angle is increased on one side. The resulting asymmetric undulatory wave leads to a change in direction. This steering principle is called the amplitude modulation method (AMM). Most researchers have used this method for the navigation of the snake robot. Sfakiotakis and Tsakiris [11] describes sensorbased control to collect information about the surroundings 
and steering of an undulatory robot through a corridor by adding an angular offset. Matsuo and Ishii [23] also applied the AMM to change the direction of a neurally controlled snake robot. Manikas [21] has summarized the methods for the turn motion of the snake robot. However, only qualitative results for the implementation of turn motion are available in previous studies. The exact relation between the change in joint angle and the turn angle is still unclear. Here, we quantitatively analyze the relation between the change in joint angle and the steering angle, proposing a general model for neurally controlled steering by AMM.

Before using AMM-based steering for collision avoidance, the snake robot's direction of motion has to be determined. A head-navigated serpentine locomotion is proposed by the neural controller. In contrast to traditional serpentine motion, the head of the head-navigated snake robot always points along the direction of movement. Using this fact, the effective distance between the obstacle and the snake robot can be calculated conveniently by means of three infrared (IR) range sensors and a camera installed on the head module. The camera takes streaming video which is then processed to find the obstacles. Based on the height of the obstacle, controller is made to decide whether to crawl over the obstacle or avoid it. The turning angle required to avoid the obstacle can be calculated using the multiple sensory signals. Using AMM method, the snake robot is made to navigate in an adaptive collision-free manner or provide sinus lifting motion for adaptive crawling.

This paper is organized as follows. Section 2 introduces an adaptive algorithm for direction collision avoidance. Section 3 gives an overall idea of the system architecture and its modules. The implementation in MATLAB is described in Section 4.The fuzzy controller to control steering of the robot is presented in Section 5. Section 6 gives an idea of real time vision processing. Finally, the work is concluded with the results obtained till present.

\section{ADAPTIVE ALGORITHM}

For the snake-like robot, the serpentine gait often also called lateral undulation is commonly used:

$$
\begin{gathered}
\varphi_{i, h}(t)=A_{h} \sin \left(\omega_{h} t+(i-1) \emptyset_{h}\right)+\gamma_{h} \\
(i=1, \ldots, n-1)
\end{gathered}
$$

Where parameters, $A_{h}, \emptyset_{h}$ determine shape of the snake and parameter $\gamma_{h}$ modifies the serpentine curve. It acts as the angular offset that provides a means for the steering mechanism. $\gamma_{h}$ is set to zero for locomotion in straight line. If $\gamma_{h}$ is non-zero the mechanism moves along a curved path, clockwise or counter clockwise, depending on the sign of $\gamma_{h}$. Links are labeled by index $i$ and $h$ which means that the gait acts in horizontal direction.
This expression is derived by an inspiration of the efficient locomotive behavior of real snakes. Since the embedded platform provides a limited computational capability, the complexity of control mechanisms are reduced to run the algorithms in real time. Other requirements for this work are related to a minimal number of parameters changed during locomotion process; such as holding the direction to the target until the target is reached and a minimum of directional changes for the energetic efficiency. This type of locomotion strategy is motivated by possible scenarios in hazardous and dangerous areas, where the robot should optimize its own behavior for a fast coverage of the targeted area.

Since a snake-like robot is not able to change rapidly its direction of movement, the gait planning and adaptation should be iteratively performed.

The adaptive algorithm for collision avoidance is applicable only for $2 \mathrm{D}$ locomotion. In order to transfer this idea to a 3D case scenario, gaits that allow movement of the snake in a 3D environment are also investigated. In [1], an overview of different possible gait formulations and locomotion strategies in planar as well as 3D environment are summarized. One of the promised gaits for a 3D locomotion is the combination of lateral undulation gait with an additional sinusoidal generator that controls the vertical gait.

Therefore, an additional parameter $\emptyset_{0}$ is required to control the phase difference between the horizontal and vertical gait:

$$
\begin{gathered}
\varphi_{i, v}(t)=A_{v} \sin \left(\omega_{v} t+(i-1) \emptyset_{v}+\emptyset_{0}\right)+\gamma_{v} \\
(i=1, \ldots, n-1)
\end{gathered}
$$

Where, index $v$ means the vertical direction. Applying only the vertical gait to the snake will produce only a caterpillarlike gait. Combination of both is required to create twist-like movement patterns that resemble a real snake.

\section{SYSTEM ARCHITECTURE}

The overall system architecture is described here. Each of the following sections gives a brief idea on each module and its functions. 


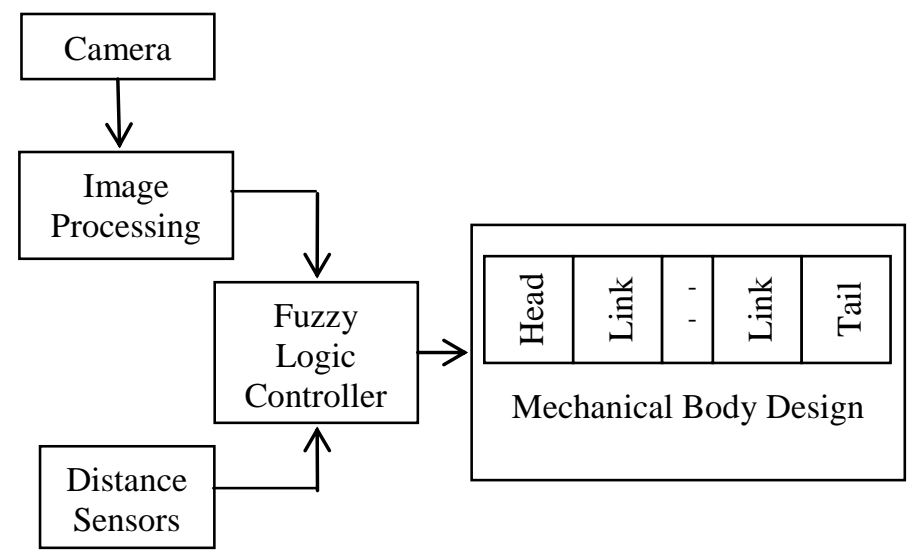

Fig.1.System Architecture

The input to the system comprises of a streaming video from a camera installed on the head of the snake robot and sensory signals placed along with the camera and on two sides of the robot body. The video gives an account of the environment in which the snake robot is moving. It captures the scene at particular instants of time and then undergoes processing to detect the height of the obstacles in the scene. The distance sensors provide the distance of the robot's body to the obstacles. This information gives an idea of whether the obstacle in the video is nearby or far away. Integration of multiple distance sensors alongside the body of the snake robot improves the collision avoidance strategy while it has to undergo an angular deflection in its path.

The input parameters are then fed to a fuzzy logic controller which provides the controller action to the snake robot. The mechanical structure of the snake robot is designed by using SimMechanics in Simulink and the controller action is supplied to the actuators that provide motion to the body links. The Matlab-Simulink platform shows the simulation results of animation of the snake robot along with the image processing GUI for obstacle detection.

\section{MATLAB IMPLEMENTATION}

For studying the dynamics of the snake-like topology, Matlab Simulink with the combination of the SimMechanics toolbox is used. SimMechanics uses the second order Euler-Lagrange dynamic equations for calculation of dynamics and allow efficient and fast multi-body simulation. The snake robot is modeled following the idea that modules are connected to the ground by six DOF joints. This allows each module to translate and rotate along all six axes in space. An arrangement is made to simulate friction and body contact with the ground.

A spring-damper system (Eq. 3) is used to model the normal forces during the ground contacts.
To simulate the hardness of the ground the spring coefficient $k$ is set to a rather high value. The damping coefficient $d$ is smaller in contrast to $k$ and is necessary to damp out the oscillations.

$$
F_{n}^{i}=-k p_{z}^{i}-d v_{z}^{i}, \quad \forall p_{z}^{i}<0
$$

Where, $i$ denote the link number and $p_{z}^{i}$ is the position and $v_{z}^{i}$ is the velocity component in $\mathrm{z}$-direction.

To model these frictions and simultaneously not to overload the simulation, we use the most simple friction model in both planar directions:

$$
F_{R_{l}}^{i}=\mu_{t} F_{n}^{i} \text { and } F_{R_{t}}^{i}=\mu_{n} F_{n}^{i}
$$

Where indexes $l$ and $t$ denote longitudinal (tangential) and transversal (normal) directions. Here viscose friction or slipstick effects are not considered because the mass and the speed of the snake links is quite low and hence these parts can be neglected at the current state.

\section{FUZZY LOGIC CONTROLLER}

Fuzzy logic controller (FLC) is used to control the navigation of the snake robot. It has a simple structure and one of the most important and interesting characteristic of a fuzzy logic controller is that linguistic rules can be used to control any complicated system.

In the case of the snake robot, here three simple rules are derived that can be used as a rule base of FLC. As mentioned in section 2, parameter $\gamma_{h}$ in Eq. 1 provides a means for steering mechanism of the snake robot. When $\gamma_{h}$ is zero, the snake moves in a straight line. When $\gamma_{h}$ is positive, it turns left in a circular path and when it has a negative value it turns right. This is because the increasing absolute value of $\gamma_{h}$ results in a decrease in the radius of circular path. In other words, when $\gamma_{h}$ is high the snake robot turns sharply to the left or right depending on sign of $\gamma_{h}$. This sharp turn results in singular configuration of snake robot that is not desirable. Therefore, selection of optimum value of $\gamma_{h}$ is to be a compromise between speed of turning and singularity avoidance.

The proposed FLC controls direction of motion of the snake robot based on sensed information. Input to the FLC is taken to be the difference between orientation of snake robot and desired orientation $\left(\Delta \xi=\xi^{*}-\xi\right)$.

As robot orientation changes during locomotion, sinusoidally, an average of links orientation is selected as a measure for the direction of the whole robot. This is shown in Figure 2

$$
\xi:=\frac{1}{n} \sum_{i=1}^{n} \theta_{i}
$$




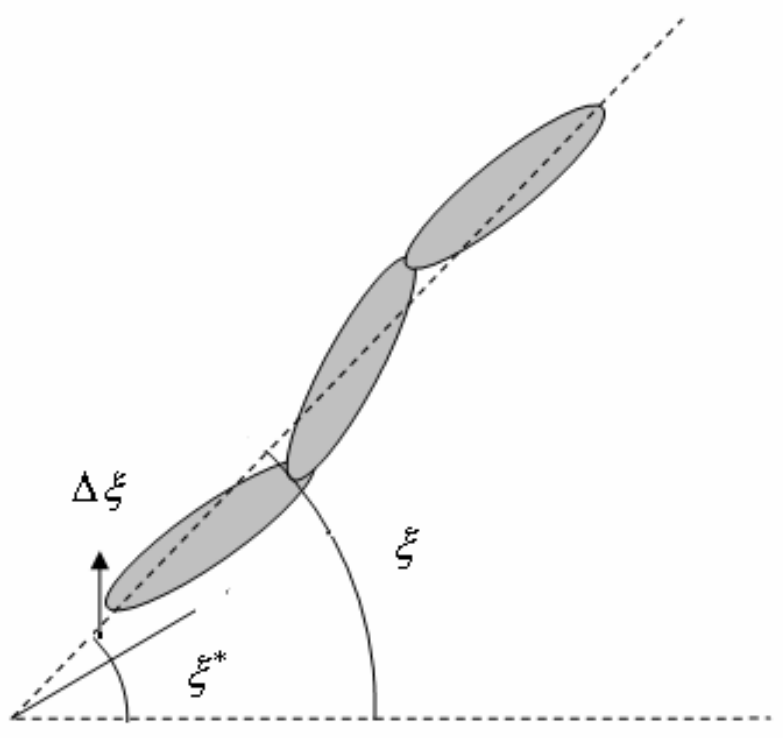

Fig.2. Direction of whole system and desired orientation

Three input membership functions $\mathrm{P}$ (positive), $\mathrm{Z}$ (zero) and $\mathrm{N}$ (negative) are proposed here (Fig. 2). The output of FLC is steering parameter $\gamma$. Three output membership functions $P$ (positive), $\mathrm{Z}$ (zero) and $\mathrm{N}$ (negative) are defined. The parameters are tuned by trial and error base on a compromise between speed and singular configuration avoidance. Figure 3 shows the output and input membership functions.

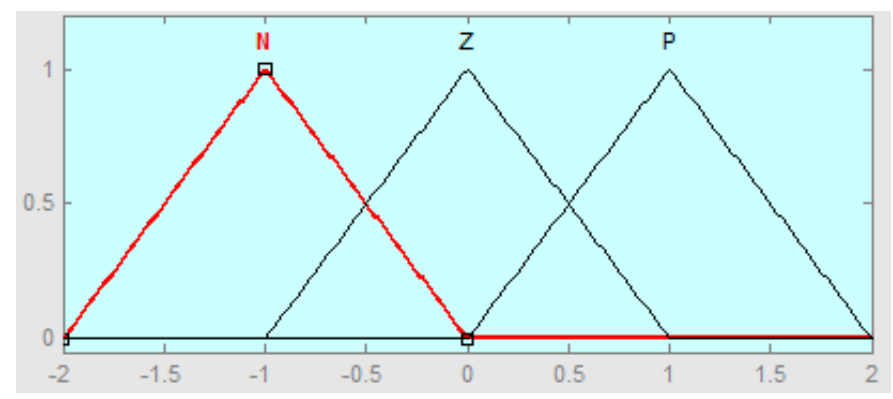

Output Membership Functions

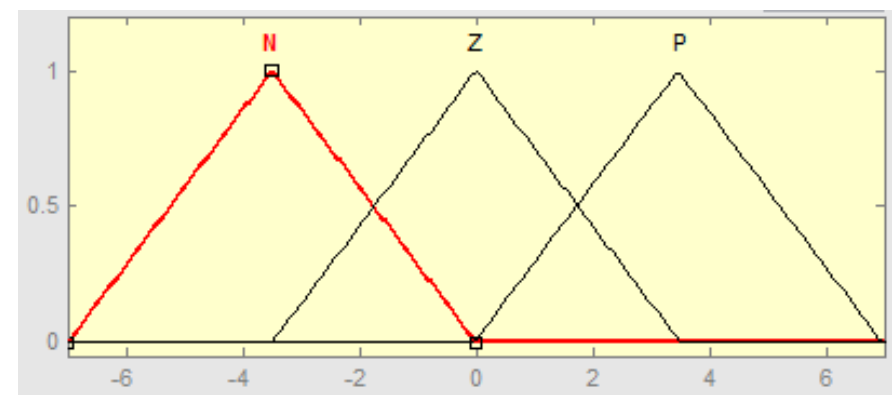

Input Membership Functions
To solve the navigation problem a model based on human knowledge is built here. The knowledge is formalized in the form of linguistic rules for navigating a snake robot to a predefined goal. An example of a linguistic rule is the following:

"If angle of snake robot direction is more than desired one, then turn left".

Utilizing these linguistic rules and relations mentioned in section 2, the rule base of FLC is built as:

"If $\Delta \xi$ is Zero then $\gamma_{h}$ is Zero.

If $\Delta \xi$ is Positive then $\gamma_{h}$ is Positive.

If $\Delta \xi$ is Negative then $\gamma_{h}$ is Negative."

Centroid method for defuzzification, product for AND operator and maximum for OR operator is chosen for the proposed FLC.

The position of the fuzzy controller in the system affects the movement of the snake robot. The controller has to be placed in such a way that the input is the orientation factor of the snake robot and the output is provided to the servo actuator such that it alters the angle of motion of the snake robot.

\section{REAL TIME VISION PROCESSING}

The path chosen by the robot must be ensured to be free of obstacles. For this, their existence must be identified and their positions located.

In present scenario, a real time video is captured by the webcam integrated in the laptop. The captured vision is then processed in Matlab using Image acquisition and Image Processing toolbox. The image is acquired via Simulink file of the snake robot and called out as a GUI. The configuration of the webcam can be selected during simulation itself.

The streaming video is then processed in a number of steps to finally an image that presents the contour displaying the obstacles. The steps involved in image processing are described below:

Step 1: The video is accessed as separate color signals (RGB signals) for ease of processing.

Step 2: A level of threshold is defined for converting the video into its gray equivalent. This produces a black \& white video of the input RGB video.

Step 3: The filled contour of the video is plotted using gray color map. This converts the video into a still image.

The still image of a particular frame or instance can then be send to calculate the heights of the objects using Euclidean distance method. Based on the height of the obstacle controller action is to be provided to the robot.

Fig.3. Input and Output Membership Functions 
If the height is less than or equal to the overall height of the snake robot, the robot is made to crawl over the obstacle. Otherwise, proper angle are given to the servo motors to turn the snake away from the obstacle but retaining its path to the target.

Recognition of the target can also be done using image processing techniques for shape recognition. It is to be ensured that the processing time be of a reasonable value such that the robot capture the next frame and make its decision in an effective manner

\section{CONCLUSIONS}

The use of snake-like robots has increased dramatically for rescue and surveillance purposes. Though various methodology and techniques have been described in currently used robots, most of them are assigned for a particular operation only. i.e., snake-like robots are designed as application oriented systems. A hybrid model of such a system will prove to be an added gem to robotics application.

An analysis about the type of techniques to be used in the proposed system has been decided. The decision was made based on studies conducted over previous researches. The resulting analysis showed that various techniques and analysis methods have been conducted for the implementation of snake like robot. Most of the analysis was done for application of a particular purpose. Here an effort is done to implement a hybrid model capable of adaptive locomotion. This is employed by integrating various techniques and analysis methods into a single prototype.

The most advantageous technique was to integrate a real time vision processing system along with the sensory systems that are being recently. An effort was taken to provide processing techniques to streaming video to produce an image per frame that gives a map indicating the position of obstacles.

A Simulink based 3D model of snake was also developed using its mechanical constraints. The motion of which can be controlled by the angle given to the servo actuators.

\section{REFERENCES}

[1]. A. Transeth, .K. Pettersen and P.Liljebäck. "A survey on snake robot modeling and locomotion" In Robotica, 27(7), Cambridge University Press, 2009, pp. 999-1015

[2]. Anscombe, R., Bryant, A., Buckingham, R., Ferguson, G., Graham, A., Lichon, M., \& Parry, N. "Snake-arm Robots Conduct Nuclear Maintenance", IYNC, Finland, 2006.

[3]. Bernhard Klaassen, Karl L. Paap, IEEE INTERNATIONAL CONFERENCE ON ROBOTICS \& AUTOMATION, Detroit, 1999, Vol. 4.
[4]. Danfeng Zhang, Chengdong Wu, Bin Li, "Study on the Optimal Passive Creeping Control of the Snakelike Robot", IEEE CONFERENCE ON INTELLIGENT CONTROL \& AUTOMATION, JULY 6-8 ,2012, Pg. 99-103

[5]. Eugen Meister, Sergej Stepanenko, and Serge Kernbach, "Adaptive Locomotion Of Multibody Snake-Like Robot", MULTIBODY DYNAMICS 2011, ECCOMAS Thematic Conference, July 4-7, 2011.

[6]. Frederic Boyer and Shaukat Ali, "Recursive Inverse Dynamics of Mobile Multibody Systems With Joints and Wheels", IEEE TRANS. ON ROBOTICS, APRIL 2011, Vol. 27, No. 2, Pg. 215-228

[7]. Frederic Boyer, Shaukat Ali, and Mathieu Porez, "Macro continuous Dynamics for Hyper redundant Robots: Application to Kinematic Locomotion Bio inspired by Elongated Body Animals", IEEE TRANS. ON ROBOTICS, APRIL 2012, Vol. 28, No. 2, Pg. 303-317

[8]. Gibson, T. "Army technology expands snakerobotics". July 23, 2010. Retrieved from http://www.army.mil/-news/

[9]. Hiroaki Fukushima, Shogo Satomura, Toru Kawai, Motoyasu Tanaka, Tetsushi Kamegawa and Fumitoshi Matsuno, "Modeling and Control of a Snake-Like Robot Using the Screw-Drive Mechanism", IEEE TRANS. ON ROBOTICS, JUNE 2012, Vol. 28, No. 3, Pg. 541-554.

[10]. Michele Guarnieri, I. Takao, E. F. Fukushima, and Shigeo Hirose, "HELIOS VIII: Toward Practical Robots for Search and Rescue Operations," JOURNAL OF ROBOTICS AND MECHATRONICS, 2008. Vol. 20, No. 5, Pg. 675694.

[11]. M. Sfakiotakis and D. P. Tsakiris, "Biomimetic centering for undulatory robots," The Int. J. Robot. Res., 2007, Vol. 26, Nos. 11-12, PP. 1267-1282.

[12]. Pal Liljebäck, Idar U. Haugstuen, and Kristin Y. Pettersen, "Path Following Control of Planar Snake Robots Using a Cascaded Approach", IEEE TRANS. ON CONTROL SYSTEMS TECHNOLOGY, JAN 2012, Vol. 20, No. 1, Pg. 111-126

[13]. Pal Liljebäck, Kristin Y. Pettersen, Øyvind Stavdahl and Jan Tommy Gravdahl, "Experimental Investigation of Obstacle-Aided Locomotion With a Snake Robot", IEEE TRANS. ON ROBOTICS, AUGUST 2011, Vol. 27, No. 4, Pg. 792-800

[14]. Pal Liljebäck, Kristin Y. Pettersen, Øyvind Stavdahl and Jan Tommy Gravdahl, "Controllability and Stability Analysis of Planar Snake Robot Locomotion", IEEE TRANS. ON AUTOMATIC CONTROL, JUNE 2011, Vol. 56, No. 6, Pg. 13651380.

[15]. Pal Liljeback, Kristin Y. Pettersen, Oyvind Stavdahl, and Jan Tommy Gravdahl, "Snake Robot 
Locomotion in Environments With Obstacles", IEEE/ASME TRANS. ON MECHATRONICS, DEC 2012, Vol. 17, No. 6, Pg. 1158-1169

[16]. S. Hasanzadeh A.-R. Akbarzadeh-T. M.-R. Akbarzadeh-T, "Fuzzy Meta-Level Control of Snake Robots", First Joint Congress on Fuzzy and Intelligent Systems, FERDOWSI UNIVERSITY OF MASHHAD, Iran, Aug 29-31, 2007.

[17]. S. Hirose and E. F. Fukushima, "Snakes and Strings: New Robotic Components for Rescue Operations," THE INTERNATIONAL JOURNAL OF ROBOTICS RESEARCH, 2004. Vol. 23, No. 4-5, Pg. 341-349.

[18]. Satoshi Toyoshima, Motoyasu Tanaka and Fumitoshi Matsuno, "A Study on Sinus-Lifting Motion of a Snake Robot With Sequential Optimization of a Hybrid System", IEEE TRANS. ON AUTOMATION SCIENCE AND ENGINEERING, JUNE 19, 2013, Vol. PP, No. 99, Pg. 1-6

[19]. Science Daily. "Snake-Like Robot And Steady-Hand System Could Assist Surgeons." Dec 25, 2006. Retrieved from http://www.sciencedaily.com/

[20]. Thomas, K. K. "Robotics in Biomemics-Snake Robot", Nov 2, 2008.

[21]. T. W. Manikas, K. Ashenayi, and R. L. Wainwright, "Genetic algorithms for autonomous robot navigation,” IEEE Instrum. Meas. Mag., Dec 2007, Vol. 10, No. 6, PP. 26-31.

[22]. Xiaodong wu and shugen ma, "Neurally controlled steering for collision-free behavior of a snake robot", IEEE TRANS, 2013, Vol. PP, No. 99, Pg. 1

[23]. X. Wu and S. Ma, "CPG-based control of serpentine locomotion of a snake-like robot," Mechatronics, 2010, Vol. 20, No. 2, PP. 326-334.

\section{BIOGRAPHIES}

Blessy Mariam Markose received her B.Tech degree in Applied Electronics \& Instrumentation from M.G University, Kerala, India, in 2012. She is currently doing her M.Tech in Process Control \& Instrumentation at Hindustan University, Chennai, India. Her Master's project is based on control and implementation of an intelligent snake robot. Her areas of interests are robotics, automation, control systems design etc.

Harshitha loke received her M.Tech degree in Process Control\& Instrumentation from National Institute of Technology, Tiruchirapalli, India in 2010. She is currently working in Hindustan University, Chennai, India. Her areas of interest are advanced process control, control design, robotics and automation. She has worked with controller design for unstable time delay systems using Pade's approximation. She worked with controller design for Inverted Pendulum and integrating process (liquid level tank).Currently she is working with intelligent snake robot. 